\title{
BMJ Open Acceptance, adherence and dropout rates of individuals with COPD approached in telehealth interventions: a protocol for systematic review and meta-analysis
}

\author{
Saeed Mardy Alghamdi, ${ }^{1,2}$ Tania Janaudis-Ferreira, ${ }^{1,3}$ Rehab Alhasani, ${ }^{1,4,5}$ \\ Sara Ahmed ${ }^{1,3,4}$
}

To cite: Alghamdi SM, Janaudis-Ferreira T, Alhasani R, et al. Acceptance, adherence and dropout rates of individuals with COPD approached in telehealth interventions: a protocol for systematic review and meta-analysis. BMJ Open 2019;9:e26794. doi:10.1136/ bmjopen-2018-026794

- Prepublication history and additional material for this paper are available online. To view these files, please visit the journal online (http://dx.doi. org/10.1136/bmjopen-2018026794).

Received 19 September 2018 Revised 18 December 2018 Accepted 25 January 2019
Check for updates

(C) Author(s) (or their employer(s)) 2019. Re-use permitted under CC BY-NC. No commercial re-use. See rights and permissions. Published by BMJ.

For numbered affiliations see end of article.

Correspondence to

Dr Sara Ahmed;

sara.ahmed@mcgill.ca

\section{ABSTRACT}

Introduction Telehealth interventions have the potential of improving exacerbation and health outcomes for individuals with chronic obstructive pulmonary disease (COPD), by delivering care in between clinical visits. However, the precise impact on avoiding exacerbation and reducing the incidence of hospital readmissions remains inconclusive. This lack of knowledge on the effectiveness of telehealth for COPD care might be due to non-adherence or partial adherence to intervention programmes and/or the withdrawal of participants over the course of previous studies.

Objectives To conduct a systematic review of trials of telehealth interventions (including randomised control trials (RCT), crossover and pre-post studies) to: (1) estimate the acceptance, adherence and dropout rates; (2) identify the reasons for dropout from telehealth interventions among individuals with COPD; (3) evaluate the impact of trialrelated, sociodemographic and intervention-related factors on the acceptance, adherence and dropout rates and (4) estimate the extent to which the acceptance, adherence and dropout rates impact outcomes in comparison with usual monitoring.

Methods and analysis A systematic literature review of four databases from earliest records to November 2018 will be carried out using CINAHL, Medline (Ovid), Cochrane Library and Embase. Randomised and nonrandomised control studies will be included, in addition to crossover and pre-studies post-studies comparing telehealth with standard monitoring among individuals with COPD only. Two independent reviewers will screen all relevant abstracts and full-text studies to determine eligibility, assess the risk of bias and extract the data using structured forms. If the included studies are sufficiently homogenous in terms of interventions, populations and objectives, a meta-analysis will be performed.

Ethics and dissemination Ethical considerations are not required for this research.

Trial registration number CRD42017078541.

\section{INTRODUCTION}

According to the Global Initiative for Chronic Obstructive Lung Disease (GOLD), chronic
Strengths and limitations of this study

- This systematic review aims to objectively estimate the acceptance, adherence and dropout rates of people with chronic obstructive pulmonary disease (COPD) enrolled in telehealth interventions, and the associated variables that potentially impact or are impacted by these rates.

- This systematic review will update existing knowledge on trial-related, patient-related and intervention-related factors potentially influencing acceptance, adherence and dropout rates.

- Exploring acceptance, adherence and dropout rates in COPD telehealth care and the associated factors will allow future researchers to design prospective clinical trials, while increasing the validity and generalisability of their results.

- The exclusion of papers written in languages other than English might leave relevant studies out of the review.

obstructive pulmonary disease (COPD) is a common disease characterised by the persistent limitation of airflow to the lungs. It can be prevented and treated; furthermore, it is progressive in nature and associated with enhanced chronic inflammatory responses (in the airways and lungs) to noxious gases. ${ }^{1}$ Airflow limitations in COPD can lead to respiratory exacerbation which is defined as an acute worsening of respiratory symptoms. ${ }^{1}$ Exacerbations can negatively impact an individual's health status, often resulting in hospitalisation. ${ }^{23}$ COPD is a major public health problem, and individuals with COPD require appropriate management strategies to minimise the likelihood of hospitalisation. ${ }^{4}$

Telehealth refers to the use of electronic information and communication technologies to support distance healthcare, which allows healthcare professionals and 


\begin{tabular}{|c|c|}
\hline Terminology & Definitions \\
\hline Telehealth & $\begin{array}{l}\text { Using electronic information and } \\
\text { communication technologies to support } \\
\text { distance healthcare, which allows } \\
\text { healthcare professionals and long- } \\
\text { distance patients to exchange information } \\
\text { and support access to healthcare } \\
\text { services. }^{5}\end{array}$ \\
\hline Telemonitoring & $\begin{array}{l}\text { Using electronic technologies, equipment } \\
\text { and sensors to transfer clinical data } \\
\text { from patient settings to the healthcare } \\
\text { providers at the clinical settings. }{ }^{5}\end{array}$ \\
\hline Telemedicine & $\begin{array}{l}\text { Using e-health and communications } \\
\text { networks for the delivery of healthcare } \\
\text { services and medical education from one } \\
\text { geographical location to another. }\end{array}$ \\
\hline Telehomecare & $\begin{array}{l}\text { Using electronic information and } \\
\text { communication technologies to support } \\
\text { care and treatment between a patient's } \\
\text { home and professional healthcare } \\
\text { settings. }\end{array}$ \\
\hline
\end{tabular}

\begin{tabular}{|c|c|}
\hline Teleconsultation & $\begin{array}{l}\text { Using videoconferencing and webcams } \\
\text { to connect the healthcare provider with } \\
\text { patients, allowing the healthcare provider } \\
\text { to assess, diagnose, and treat patients. }\end{array}$ \\
\hline Tele-education & $\begin{array}{l}\text { Using web-based platforms to educate } \\
\text { patients about the patient disease } \\
\text { management. }\end{array}$ \\
\hline $\begin{array}{l}\text { Telehealth } \\
\text { Pulmonary } \\
\text { Rehabilitation }\end{array}$ & $\begin{array}{l}\text { Using telehealth to deliver pulmonary } \\
\text { rehabilitation to COPD patients via } \\
\text { communication technologies, and } \\
\text { maintain connections between patients } \\
\text { and healthcare professionals. }\end{array}$ \\
\hline Dropout rate & $\begin{array}{l}\text { The number of participants who dropout } \\
\text { divided by the number of participants who } \\
\text { consented to participate. }^{32}\end{array}$ \\
\hline Acceptance rate & $\begin{array}{l}\text { The number of participants who } \\
\text { consented to participate divided by the } \\
\text { number of eligible participants. }\end{array}$ \\
\hline $\begin{array}{l}\text { Adherence } \\
\text { definition }\end{array}$ & $\begin{array}{l}\text { The ability to measure telehealth use and } \\
\text { observe the intention to use telehealth } \\
\text { technology. }\end{array}$ \\
\hline
\end{tabular}

long-distance patients to exchange information and enable access to healthcare services. Various terms are used throughout the medical industry to reference specific applications and use cases for telehealth-these are presented in table $1 .^{5}$ For example, telehealth interventions with COPD could be used to deliver care and it can help to detect exacerbations at an early stage, minimising the potential for emergency admissions and facilitating self-management. ${ }^{5-8}$ Telehealth is also used for remote monitoring of a patient's clinical data, such as their vital signs; this enables healthcare teams to identify disease deterioration at an early stage and provide the requisite care in a timely manner. This has the effect of helping individuals manage their diseases and for facilitating early detection of disease exacerbation. ${ }^{9}$ There is growing evidence that telehealth may be a useful tool for minimising hospital admissions due to respiratory exacerbations, particularly in the case of individuals who are constrained by geographical barriers, or have limited access to healthcare services. ${ }^{5}{ }^{10}$ Clinical trials have shown that individuals with COPD have positive attitudes towards participating in telehealth and that telehealth can promote patients' independence toward self-management. ${ }^{11-17}$ However, the precise impact of telehealth on avoiding exacerbation and reducing hospital readmissions remains inconclusive. ${ }^{5}$ The uncertainty about the impact of telehealth may be due to non-adherence or partial adherence to intervention techniques as well as the withdrawal of participants over the course of previous studies. ${ }^{18}$ Dropout rates for telehealth vary across clinical trials. ${ }^{6}{ }^{19-22}$ It is unclear which variables are most strongly associated with non-adherence and withdrawal, although possible factors may be related to participant characteristics, intervention characteristics, and the context and environment in which the intervention is delivered. Understanding the characteristics of individuals with COPD, features of the interventions undertaken, and the environment of clinical trials is essential for reducing dropout rates in future studies. Such understanding will help with designing prospective clinical trials, while also increasing the validity and generalisability of their results. ${ }^{23}$ Evaluating the reasons that prevent individuals with COPD from enrolling and completing telehealth interventions may help clinicians appropriately tailor interventions to the individuals' needs and limit dropout rates. ${ }^{183}$ Moreover, researchers can explore individual's preferences and use them to develop more desirable and feasible telehealth interventions.

\section{OBJECTIVES}

The objectives of this systematic review are to:

1. Estimate acceptance, adherence and dropout rates in trials of telehealth interventions (including RCT, crossover and pre-studies post-studies).

2. Identify the reasons for dropout from the intervention.

3. Estimate the impact of trial-related factors, sociodemographic factors and intervention-related factors on acceptance, adherence and dropout rates.

4. Estimate the extent to which acceptance, adherence and dropout rates affect patient's outcomes.

\section{METHODS}

This systematic review will be conducted according to the Preferred Reporting Items for Systematic Review and Meta-Analysis Protocols (PRISMA-P).

\section{Patient and public involvement}

Patients and or public were not involved in this systematic review. 


\section{Inclusion criteria}

1. Study type: randomised or non-randomised control trials, observational single arm pre-post trials, and crossover clinical trials.

2. Population: studies including individuals diagnosed with COPD based on reported forced expiratory volume in one second as a percentage of predicted $\left(\mathrm{FEV}_{1} \%\right)$ will be considered for this review.

3. Type of intervention: this review includes any information technology tool designed for the clinical support of patients with COPD involving the remote exchange of data between a patient and a healthcare professional. This includes, for example, telehealth, telecare, telehomecare, e-health, telemonitoring, telerehabilitation, telemedicine, home monitoring, digital monitoring, web-based monitoring, or internet-based monitoring as part of a COPD-management plan.

4. Type of outcome: outcomes include health-related quality of life, adherence to the action plan, exacerbations, duration of hospital stay, hospitalisation or utilisation of health services (including COPD related cost), and exercise capacity.

\section{Exclusion criteria}

1. Trials not published in English.

2. Studies that do not describe the telehealth intervention researched, including delivery methods, mode of administration and frequency of data transmissions.

3. Studies that do not report the number of individuals who were approached, who gave their consent, and who dropped out.

\section{SEARCH STRATEGY}

\section{Electronic databases}

A systematic search of the following databases from earliest records to November 2018 will be undertaken to identify relevant articles: CINAHL; Medline (Ovid); Cochrane Library and Embase. The following Medical Subject Headings (MeSH terms), subject headings and keywords or combinations thereof will be used: telecare; telehomecare; telehealth; e-health; telemonitoring; telerehabilitation; telemedicine; home monitoring; digital monitoring; web-based monitoring; internet-based monitoring; Chronic Obstructive Pulmonary Disease; Chronic Obstructive lung disease and COPD. The search strategy was developed in collaboration with a health sciences librarian, to ensure the involvement of appropriate and necessary keywords in the review. Keywords and subject terms will be customised for each database. Further, all words with the prefix 'tele-' will be searched both with and without a hyphen (eg, both 'tele-monitoring' and 'telemonitoring'). The search strategies from Medline (Ovid) are presented in online supplementary appendix 1 .

\section{Manual literature search}

We will perform manual searches of reference lists of all relevant primary studies and systematic reviews to identify any additional studies that were not captured by our original search.

\section{Reference manager}

All articles will be imported to EndNote software and any duplicates removed.

\section{Search procedures}

The search will be performed by two team members (SA), after which all articles will be imported to EndNote V.7.7 and any duplicates removed. All article titles and abstracts will be screened by two independent reviewers. A manual search of the reference lists of relevant studies shall be undertaken, to identify any additional articles that were missed by the database search but that might be suitable for inclusion in the review. Subsequently a full-text review of all the included articles will be carried out. Disagreements between reviewers will be resolved through discussion. If no consensus can be reached, a third reviewer's decision will be considered. Any study that does not meet the inclusion criteria will be excluded and the reasons for exclusion recorded according to the PRISMA flowchart.

\section{Study selection and data extraction}

A data extraction form will be created using an Excel sheet. Two independent reviewers will perform the data extraction. First, reviewers will pilot the data extraction form based on ten included studies. Second, any disagreement between reviewers at this stage will be resolved by consensus. If no consensus can be reached, a third reviewer will make the decision. The first reviewer will then start extracting data. The second reviewer will check the consistency of the data and identify any errors. In case information is missing from an included study's published manuscript, its authors will be contacted and asked for clarification.

\section{Data extraction and data management}

Data related to the study characteristics, population characteristics and intervention characteristics, as defined in the intervention Complexity Assessment Tool for Systematic Reviews, shall be extracted. ${ }^{25}$

Study characteristics: authors' names; year of publication; country; research design, as well as recruitment methods.

Population characteristics: age; gender; level of education; GOLD grade and/or $\mathrm{FEV}_{1}$ as a percentage of predicted $\left(\mathrm{FEV}_{1} \%\right)$; smoking history; number of COPD patients who consented to participate, were approached, dropped out and completed the study, as well as reasons for dropout.

Intervention characteristics: settings, methods, frequency and components of telehealth (active elements, targeted behaviour, targeted users, the degree of tailoring, health professional assistance) and duration of intervention. 


\section{OUTCOMES}

All reported outcomes of COPD will be extracted, as will the effect size (ES) of telehealth intervention on these outcomes. The ES will be calculated if it is not mentioned by the author(s). ES calculation will be performed according to results from the first post-interventional evaluation, which will reflect the earliest impact of telehealth interventions on outcomes. Any results after the first post-interventional evaluation (eg, results from multiple follow-up points) will not be considered in the ES calculation. Also, the ES on the main outcome will be included in the analysis if the studies have more than one outcome.

\section{Outcomes}

Outcomes extracted from each study:

All primary and secondary outcomes defined by each study will be extracted. These include, but are not limited to:

Hospitalisation: admissions due to exacerbations and causes of hospitalisation will be reported. Attention shall be paid to differences between count and dichotomous data (eg, the count of participants in each group who experience at least one exacerbation event vs number of events per intervention group).

Exacerbation rate is a commonly reported outcome. ${ }^{26}$ As exacerbations can be reported in different ways, the data collection allows for the following numbers to be recorded: number of exacerbations or exacerbation rate (that may also be classified based on the patient disease severity), all-cause mortality and number of patients per study group who died during the survey.

Adherence to the action plan: (including any measurement mentioned by the authors to report the adherence to the action plan-for example, adherence to intervention, adherence to physiological monitoring, adherence to symptom monitoring, adherence to medication, adherence to exercise and adherence telehealth and/or telemonitoring).

Health-related quality of life: disease-specific or non-disease-specific quality of life reported by a validated instrument.

Physical activity measurements (any type reported by a validated measurement system).

Outcome of adherence for this review:

Outcomes of this review are acceptance, adherence and dropout rates. When these outcomes are not reported in the original studies, we will calculate the rates as follows:

The acceptance rate will be calculated by taking the total number of participants who accepted, agreed, and consented to participate in this study and dividing it by the number of participants who were approached for involvement in telehealth intervention. The adherence rate will be calculated as the total number of participants who completed the telehealth intervention according to the study protocol divided by the number who started the intervention. The dropout rate will be calculated as number of participants who withdrew from or did not continue with the intervention divided by the number of participants who consented to participate on the study. All rates will be presented using an overall average.

\section{Risk of bias assessment}

Two team members (SA and RA) will independently assess the risk of bias for each study included in the review; the Cochrane Collaboration Risk of Bias criteria will be used for randomised clinical trials, and Scottish Intercollegiate Guidelines Network checklist (SIGN) checklist will be used for observational studies. Reviewers will independently report justifications and comments for their decisions. A third team member will be consulted to resolve any discrepancies. The AMSTAR II tool will be used to assess the risk of bias for the systematic review.

\section{DATA ANALYSIS}

Statistical Analysis System software will be used to run regression models. Possible variables associated with rates will be categorised and tested using the univariate analysis model. Subsequently, a random effect meta regression analysis will be used to estimate the effects of the participant, study, and intervention characteristics on acceptance, adherence, dropout rates. A separate model analysis will be conducted for each rate. If we are restricted in this regard and unable to perform a meta-analysis, we will synthesise and summarise the results narratively.

\section{Dealing with missing data}

Authors will be contacted to obtain any unreported data.

\section{DISCUSSION}

This systematic review aims to objectively estimate the acceptance, adherence and dropout rates of COPD populations enrolled in telehealth and the associated variables that might affect or be affected by these rates. It will help identify the extent to which associated variables can be used for an improved design of clinical trials, to suggest the characteristics of associated target populations, and to recommend elements for inclusion in telehealth intervention to support self-management.

To the best of our knowledge, this will be the first systematic review estimating acceptance, adherence, and dropout rates of COPD populations participating in telehealth, as well as the associated factors influencing these rates. Previous systematic reviews were unable to provide information about effective elements contributing to better acceptance, adherence and dropout rates using meta-regression analysis; in contrast, the current study will try to explore elements of telehealth that impact acceptance, adherence and dropout rates. ${ }^{27}{ }^{28}$ Our systematic review will analyse the literature using meta-analysis, and in doing so provide the advantage of having an opportunity to investigate and understand the correlation between pertinent factors and acceptance, adherence and dropout rates. We will provide specific information 
about the trials' characteristics (RCTs vs non-RCTs), population characteristics (ie, mild severity vs moderate severity) and intervention characteristics (ie, primary care settings vs specialty care settings), as well as how such information may facilitate users' adherence to telehealth interventions.

Furthermore, existing evidence on acceptance, adherence and dropout rates will inform methods for designing future telehealth projects focusing on COPD. This study may also be beneficial for the management of grants for research in the field.$^{29}$ It will contribute to future research by identifying the target populations among which telehealth are accepted, and identify feasible interventions. Finally, this systematic review will help tailor technological interventions to more effectively meet the needs of COPD patients.

\section{Ethics and dissemination}

This systematic review requires no ethics approval. This research will use no confidential or personal patient data. Findings will be disseminated through publication in a peer-reviewed specific journal.

\section{Author affiliations}

${ }^{1}$ School of Physical and Occupational Therapy, McGill University, Montreal, Quebec, Canada

${ }^{2}$ Department of Respiratory Care, Umm Al-Qura University College of Applied Sciences, Makkah, Saudi Arabia

${ }^{3}$ Center for Outcome Research and Evaluation (CORE), McGill University Health Center, Montreal, Quebec, Canada

${ }^{4}$ Research Center, Centre de recherche interdisciplinaire en réadaptation, Centre de Réadaptation Constance-Lethbridge, CIUSSS du Centre-Ouest-de-d'île-de-Montréal, Montreal, Quebec, Canada

${ }^{5}$ College Of Health and Rehabilitation Sciences, Princess Nourah bint Abdulrahman University, Riyadh, Saudi Arabia

Acknowledgements The authors thank the reviewers of the manuscript for their constructive feedback.

Contributors SMA, SA, TF-J and RA developed the idea and designed the study protocol. SMA, SA designed and wrote the search strategy and the first protocol draft. SMA, SA and RA planned the data extraction and statistical analysis. SA, TF-J, RA provided critical insights. All authors have approved and contributed to the final written manuscript.

Funding The main author disclosed receipt of the following financial support for the research, authorship and/or publication of this article. This study was supported by a scholarship from Umm Al Qura University in Saudi Arabia, Sara Ahmed and Tania Janaudis-Ferreira are supported by Fonds de recherche Santé (FRQS) career award.

Competing interests None declared.

Patient consent for publication Not required.

Provenance and peer review Not commissioned; externally peer reviewed.

Open access This is an open access article distributed in accordance with the Creative Commons Attribution Non Commercial (CC BY-NC 4.0) license, which permits others to distribute, remix, adapt, build upon this work non-commercially, and license their derivative works on different terms, provided the original work is properly cited, appropriate credit is given, any changes made indicated, and the use is non-commercial. See: http://creativecommons.org/licenses/by-nc/4.0/.

\section{REFERENCES}

1. GOLD, G.I.f.C.O.L.D. Diagnosis of Disease of Chronic Airflow Limitation: Asthma, COPD and Asthma-COPD Overlap Syndrome
(ACOS): Global Initiative for Chronic Obstructive Lung Disease GOLD, 2017:18.

2. Fletcher MJ, Upton J, Taylor-Fishwick J, et al. COPD uncovered: an international survey on the impact of chronic obstructive pulmonary disease [COPD] on a working age population. BMC Public Health 2011;11:612.

3. Halbert RJ, Natoli JL, Gano A, et al. Global burden of COPD: systematic review and meta-analysis. Eur Respir J 2006;28:523-32.

4. Yang $\mathrm{F}$, Xiong ZF, Yang C, et al. Continuity of care to prevent readmissions for patients with chronic obstructive pulmonary disease: A systematic review and meta-analysis. COPD 2017:14:251-61.

5. Selzler AM, Wald J, Sedeno M, et al. Telehealth pulmonary rehabilitation: A review of the literature and an example of a nationwide initiative to improve the accessibility of pulmonary rehabilitation. Chron Respir Dis 2018;15:41-7.

6. Chau JP, Lee DT, Yu DS, et al. A feasibility study to investigate the acceptability and potential effectiveness of a telecare service for older people with chronic obstructive pulmonary disease. Int $J$ Med Inform 2012;81:674-82.

7. Cox NS, Oliveira CC, Lahham A, et al. Pulmonary rehabilitation referral and participation are commonly influenced by environment, knowledge, and beliefs about consequences: a systematic review using the Theoretical Domains Framework. J Physiother 2017:63:84-93

8. Paré G, Jaana M, Sicotte C. Systematic review of home telemonitoring for chronic diseases: the evidence base. J Am Med Inform Assoc 2007:14:269-77.

9. Crist TM, Kaufman SB, Crampton KR. Home telemedicine: a home health care agency strategy for maximizing resources. Journal of Home Health Care Practice 1996;8:1-9.

10. McKinstry $\mathrm{B}$. The use of remote monitoring technologies in managing chronic obstructive pulmonary disease. QJM: An International Journal of Medicine, 2013:106;883-5.

11. Almojaibel A. Delivering pulmonary rehabilitation for patients with chronic obstructive pulmonary disease at home using telehealth: A review of the literature. Saudi Journal of Medicine and Medical Sciences 2016;4:164.

12. Burkow TM, Vognild LK, Østengen G, et al. Internet-enabled pulmonary rehabilitation and diabetes education in group settings at home: a preliminary study of patient acceptability. BMC Med Inform Decis Mak 2013;13:33

13. Holland AE, Hill CJ, Rochford P, et al. Telerehabilitation for people with chronic obstructive pulmonary disease: feasibility of a simple, real time model of supervised exercise training. $J$ Telemed Telecare 2013;19:222-6.

14. Paneroni M, Colombo F, Papalia A, et al. Is telerehabilitation a safe and viable option for patients with copd? A feasibility study. COPD 2015;12:217-25.

15. Tabak M, Brusse-Keizer M, van der Valk $\mathrm{P}$, et al. A telehealth program for self-management of COPD exacerbations and promotion of an active lifestyle: a pilot randomized controlled trial. Int J Chron Obstruct Pulmon Dis 2014;9:935.

16. Zanaboni $P$, Lien LA, Hjalmarsen A, et al. Long-term telerehabilitation of COPD patients in their homes: interim results from a pilot study in Northern Norway. $J$ Telemed Telecare 2013;19:425-9.

17. Pinnock $\mathrm{H}$, et al. P33 'Light Touch'Telemonitoring For People With COPD. Lothian: A Pilot Evaluation With Nested Qualitative Study: BMJ Publishing Group Ltd, 2014.

18. Cruz J, Brooks D, Marques A. Home telemonitoring in COPD: A systematic review of methodologies and patients' adherence. Int $J$ Med Inform 2014;83:249-63.

19. Antoniades NC, Rochford PD, Pretto JJ, et al. Pilot study of remote telemonitoring in COPD. Telemed J E Health 2012;18:634-40.

20. $\mathrm{Kim} \mathrm{J,} \mathrm{Kim} \mathrm{S,} \mathrm{Kim} \mathrm{H,} \mathrm{et} \mathrm{al.} \mathrm{Acceptability} \mathrm{of} \mathrm{the} \mathrm{consumer-centric}$ u-health services for patients with chronic obstructive pulmonary disease. Telemed J E Health 2012;18:329-38.

21. Sund ZM, Powell T, Greenwood R, et al. Remote daily real-time monitoring in patients with COPD - a feasibility study using a novel device. Respir Med 2009;103:1320-8.

22. Trappenburg JC, Niesink A, de Weert-van Oene GH, et al. Effects of telemonitoring in patients with chronic obstructive pulmonary disease. Telemed J E Health 2008;14:138-46.

23. Rozenfeld Y, Hunt JS. Effect of patient withdrawal on a study evaluating pharmacist management of hypertension. Pharmacotherapy 2006;26:1565-71.

24. Wittes J. Missing inaction: preventing missing outcome data in randomized clinical trials. J Biopharm Stat 2009;19:957-68.

25. Lewin S, Hendry M, Chandler J, et al. Assessing the complexity of interventions within systematic reviews: development, content and 
use of a new tool (iCAT_SR). BMC Med Res Methodol 2017;17:76.

26. Cazzola M, MacNee W, Martinez FJ, et al. Outcomes for COPD pharmacological trials: from lung function to biomarkers. Eur Respir $J$ 2008;31:416-69.

27. Almojaibel AA. Delivering pulmonary rehabilitation for patients with chronic obstructive pulmonary disease at home using telehealth: A review of the literature. Saudi J Med Med Sci 2016;4:164-71.

28. Cruz J, Brooks D, Marques A. Home telemonitoring in COPD: a systematic review of methodologies and patients' adherence. Int $J$ Med Inform 2014;83:249-63.

29. Maeder A, Poultney N, Morgan G, et al. Patient compliance in home-based self-care telehealth projects. J Telemed Telecare 2015;21:439-42.
30. Sood S, Mbarika V, Jugoo S, et al. What is telemedicine? A collection of 104 peer-reviewed perspectives and theoretical underpinnings. Telemed J E Health 2007;13:573-90.

31. Huniche L, Dinesen B, Grann O, et al. Empowering patients with COPD using Tele-homecare technology. Stud Health Technol Inform 2010;155:48-54.

32. Arafah AM, Bouchard V, Mayo NE. Enrolling and keeping participants in multiple sclerosis self-management interventions: a systematic review and meta-analysis. Clin Rehabil 2017;31:809-23.

33. Sieverink F, Kelders SM, van Gemert-Pijnen JE. Clarifying the concept of adherence to ehealth technology: Systematic review on when usage becomes adherence. J Med Internet Res 2017;19:e402. 